\title{
Espressione dell'approssimazione tra spagnolo e italiano: il caso di venir siendo e venir a ser
}

\author{
Maria Vittoria Ambrosini ${ }^{1}$
}

Ricevuto: 31 gennaio 2019 / Modificato: 03 giugno 2020 / Accettato: 15 giugno 2020

Riassunto. Questo studio vuole offrire un'opportunità di riflessione interlinguistica sull'espressione dell'approssimazione tra spagnolo e italiano a partire dalle due perifrasi spagnole venir siendo e venir a ser. Queste due perifrasi, infatti, sono tipicamente utilizzate dagli ispanofoni per esprimere approssimazione, la quale, a seconda del contesto, può essere di ordine quantitativo o tradursi in relativizzazione-eufemizzazione o ancora in espressione di dubbio rispetto a quanto il parlante afferma. In questo studio, si analizzano innanzitutto i punti in comune e le divergenze che caratterizzano le diverse sfumature di approssimazione - spesso talmente sottili da generare dubbi presso gli stessi ispanofoni - che possono veicolare venir siendo e venir a ser; si passa poi a un'analisi di tipo contrastivo tra spagnolo e italiano che mostrerà l'indisponibilità di perifrasi italiane corrispondenti a venir siendo e venir a ser in termini di forma e di espressione della valenza modale approssimativa. Infine, tramite un test ad hoc sottoposto a un gruppo di studenti di spagnolo italofoni, si rifletterà sia sul grado di trasparenza interpretativa che presentano per loro le due perifrasi spagnole in questione - a partire da cinque diversi contesti d'uso proposti- sia sulle soluzioni linguistiche che offrirebbero gli informanti per renderle nella loro lingua madre.

Parole chiave: perifrasi; modalità approssimativa; studio contrastivo; spagnolo; italiano.

\section{[en] Expression of approximation between Spanish and Italian: the case of venir siendo and venir a ser}

\begin{abstract}
This research aims to provide an interlinguistic in-depth analysis on the expression of approximation comparing Spanish and Italian constructions starting from two Spanish periphrases: venir siendo and venir a ser. In fact, such periphrastic structures are typically used by Spanish-speakers to express approximation and, based on its context, approximation can be quantitative or conveyed through relativization / euphemism, or by expressing doubt about what the speaker is stating. Firstly, the study focuses on the common and divergent features which distinguish the different nuances of approximation - often so subtle they may lead to confusion even among Spanish-speakers - when using venir siendo and venir a ser. Secondly, a contrastive analysis between Spanish and Italian shows the lack of Italian periphrases which are equivalent to venir siendo and venir a ser as to form and expression of the approximate modal value. Finally, a specifically-designed test - submitted to Italian speaking students learning Spanish - reveals the level of interpretative transparency that the two Spanish periphrases allow in five different contexts; through this test, informants also had the possibility to give linguistic solutions to translate the two Spanish periphrases into their native language.
\end{abstract}

Keywords: periphrases; approximate mode; contrastive linguistics; Italian; Spanish.

Universidad Autónoma de Barcelona (UAB). Departamento de Filología Francesa. Dirección de contacto: Carrer Freixures 25, 2-2, 08003, Barcelona.

E-mail: ambromavi@yahoo.it. 
Sommario: 1. Introduzione 2. Venir + gerundio e venir $+a+$ infinito: i due processi di grammaticalizzazione 2.1. Venir + gerundio: la perifrasi aspettuale 2.2. Venir + gerundio oltre la tappa aspettuale: la perifrasi modale-approssimativa 2.3. La perifrasi modale venir $+a+$ infinito 2.4. Venir + gerundio o venir $+a+$ infinito? Una questione di approssimazione 3. Venir siendo, venir a ser... e in italiano? 3.1. Venir siendo > venir essendo* 3.2. Venir a ser > venire $a(d)$ essere 4. Dallo spagnolo all'italiano: un test... approssimativo 4.1. Il test: item, obbiettivi e gruppo di informanti 4.2. Risultati 5. Conclusioni.

Come citare: Ambrosini, Maria Vittoria (2020): «Espressione dell'approssimazione tra spagnolo e italiano: il caso di venir siendo e venir a ser», Cuadernos de Filología Italiana, 27, pp. 9-30.

\section{Introduzione}

Certo, italiano e spagnolo sono lingue affini. È una realtà che sotto il punto di vista didattico tocchiamo con mano ogni volta che entriamo in classe. Una realtà che però non si compone di sole luci, ma anche di intriganti zone d'ombra, che forse, proprio perché tali, rendono tanto avvincente la comparazione tra queste due lingue. Una di queste (molte) zone d'ombra che ancora chiedono a gran voce di essere esplorate, ad esempio, riguarda le perifrasi spagnole venir siendo e venir a ser, utilizzate in spagnolo per esprimere un'ampia gamma di valori modali.

Nella prima parte di questo approfondimento, si illustrerà come si tratti di due perifrasi che non trovano una corrispondenza diretta in lingua italiana.

Ecco che la vicinanza linguistica si fa divergenza, richiamando a gran voce la nostra attenzione, sul piano contrastivo: come reagirà un ipotetico apprendente di spagnolo italofono, confrontandosi alla molteplicità di sfumature modali che venir siendo e venir a ser possono attualmente veicolare? Gli risulteranno trasparenti, opache, $o$ addirittura oscure?

Il secondo passaggio di questa riflessione interlinguistica prevede poi un focus sul come queste sfumature potrebbero essere trasmesse, da un ipotetico apprendente di spagnolo italofono, nella sua lingua madre.

Per iniziare a raccogliere qualche dato nello spirito di questa indagine - che in futuro aspira ad assumere più ampie proporzioni - si è creato un test $a d$ hoc che andasse a sondare proprio questi interrogativi, presso un gruppo di studenti di spagnolo italofoni selezionati presso la Scuola Superiore di Lingue Moderne per Interpreti e Traduttori (SSLMIT) dell'Università degli Studi di Trieste.

Pur provenendo da un gruppo di informanti con un livello di spagnolo piuttosto avanzato -anzi, forse proprio per questo- i risultati di questo breve test dimostrano che l'interpretazione delle due perifrasi spagnole di partenza non deve affatto essere data per scontata; al contrario, richiede un'attenzione speciale da parte -prima ancora che dell'apprendente italofono- del docente, che dovrà guidarlo alla scoperta e decodifica delle stesse, nella molteplicità di contesti in cui ricorrono nella lingua spagnola attuale.

\section{Venir + gerundio e venir $+a+$ infinito: i due processi di grammaticalizzazione}

Partiamo innanzitutto da un excursus più generale che ci permetta di far luce sulle perifrasi spagnole venir + gerundio e venir $+a+$ infinito: due perifrasi votate -almeno ori- 
ginariamente-all'espletazione di funzioni linguistiche piuttosto diverse ma che i rispettivi percorsi di grammaticalizzazione hanno portato ad un progressivo accostamento.

\subsection{Venir + gerundio: la perifrasi aspettuale}

Secondo Yllera (1980: 71), venir è stato l'ultimo tra i verbi intransitivi di movimento ad esordire in spagnolo come ausiliare nella formazione di perifrasi gerundivali.

La perifrasi sarebbe stata scarsamente utilizzata durante il Medioevo: in quest'epoca, invece, è ir + gerundio la perifrasi gerundivale utilizzata con maggior frequenza, cui venir + gerundio si avvicina molto come perifrasi temporale progressiva; a partire dal XIII secolo, di fatti, venir + gerundio inizierà ad essere utilizzata alternativamente a $i r+$ gerundio con questa valenza.

Nella prosa del XVI secolo, si riscontrano i primi esempi d'uso della perifrasi accompagnata da una determinazione temporale, a segnalare che l'azione è iniziata in un momento del passato e continua a svilupparsi nel presente (Spaulding 1926: 260).

A partire dal XIX secolo, la perifrasi assume infine una terza valenza (Spaulding 1926: 261): in alcune sue occorrenze, la perifrasi non segnalerebbe una progressione temporale verso il momento di osservazione, quanto piuttosto un avvicinamento fisico al soggetto, rappresentato dal pronome me o nos (es. No me vengas diciendo).

La perifrasi venir + gerundio, seguendo questa linea, si afferma quindi progressivamente come una manifestazione morfologica della cosiddetta Retrospektive Schau (Dietrich 1973 [1983: 208-209]), ossia quella prospettiva attraverso la quale il processo azionale viene inquadrato dal momento iniziale -che precede il momento di osservazione- al momento di osservazione stesso ${ }^{2}$.

Sánchez Montero (1993: 117) sottolinea che, tendenzialmente, la perifrasi attribuisce al processo azionale designato un certo matiz de insistencia o repetición. Effettivamente, come osservano Fente, Fernández y Feijóo (1976: 55), se questa perifrasi è accompagnata dell'avverbio aún, esplicito o implicito, sarebbe sostanzialmente equivalente alla perifrasi iterativa seguir + gerundio:

(1) La experiencia nos viene (=sigue) demostrando que el hombre es un animal de costumbres (Sánchez Montero 1993: 117)

Questa perifrasi può altresì presentare valore frecuentativo (Musto e Ripa 2005: 418): in tal caso, si combina con le preposizioni desde e hasta. Come osservano infatti Gómez Torrego (1988: 168) e Sánchez Montero (1993: 119), venir + gerundio può, in alcuni casi, sostituire la perifrasi continuativa llevar + gerundio:

(2) Hace tres años que vengo / llevo aconsejándote lo mismo (Sánchez Montero 1993: 119)

Tuttavia, come osserva Camus Bergareche (2004: 558), contrariamente a llevar + gerundio, venir + gerundio può anche non essere accompagnata dal complemento circostanziale che segnala l'inizio dell'azione, il quale può essere implicito nel contesto della situazione comunicativa. Tale caratteristica è probabilmente attribuibile 
alle peculiarità lessicali dell'ausiliare venir.

$\mathrm{Su}$ questa tipizzazione di venir + gerundio come perifrasi aspettuale pare dunque esistere un certo consenso in termini di studi di settore, alla base dei quali vi è appunto l'idea di base secondo la quale l'ausiliare venir avrebbe dunque subito una ricategorizzazione dei suoi valori lessicali inziali -ascrivibili a una dimensione eminentemente spaziale- in valori di ordine aspettuale, all'interno della nuova perifrasi (aspettuale, per l'appunto) venir + gerundio (Melis 2006: 878).

È interessante notare che, benché generalmente l'ausiliare venir sia incompatibile con i verbi di stato, esso li accetta nei casi in cui l'argomento del predicato sia un gruppo nominale al plurale (RAE 2009: 550), ossia nei casi in cui «el conjunto designado pueda distribuirse idealmente»:

(3) A derecha y a izquierda, los montes que a lo largo de toda la ribera venían flanqueando las choperas y los prados [...] (RAE 2009: 550)

(4) La página editorial de este diario viene siendo muy crítica desde las elecciones (RAE 2009: 550).

Il secondo degli esempi offerti dal Diccionario della RAE è particolarmente significativo: benché il predicato sia-grammaticalmente parlando- singolare, è evidente che in questo caso non si fa riferimento ad un solo editoriale, quanto piuttosto ad una serie di editoriali, considerati nella loro evoluzione in un determinato arco di tempo; così come, nell'esempio (3), si considera una serie di monti che si dispongono nella mente della persona osservante come una serie di punti progressivamente distribuiti nel suo orizzonte visivo. Il superamento di questa limitazione avviene nel momento in cui l'ausiliare venir avanza lungo il suo percorso di grammaticalizzazione, affrancandosi sempre più dalle limitazioni che lo caratterizzavano sul piano spaziale, data la sua valenza deittica originale.

\subsection{Venir + gerundio oltre la tappa aspettuale: la perifrasi modale-approssimativa}

Secondo Melis (2006: 877-879) la trasformazione di un verbo di movimento in verbo ausiliare non avviene in modo puntuale, ma per fasi consecutive di evoluzione del verbo stesso lungo l'asse di grammaticalizzazione, fino a portarlo ad un ideale stadio finale in cui esso diviene ausiliare pieno.

Infatti, come osserva l'autrice, in spagnolo esistono perifrasi che occupano posizioni diverse lungo quest'asse di evoluzione. Tutti i verbi che formano perifrasi, spiega, iniziano innanzitutto a riconvertire il loro valore deittico-spaziale iniziale in valore aspettuale-temporale; alcuni verbi, poi, proseguono la loro evoluzione passando dal piano dell'aspetto al piano della modalità: in questa casistica, rientrano per l'appunto alcuni usi di verbi spagnoli quali llegar, venir e ir.

In questa terza fase, si realizza una sorta di passaggio dall'aspetto (categoria oggettiva) ad una dimensione di approccio alla realtà piuttosto rapportata alla prospettiva del soggetto osservante: la perifrasi subisce nuove alterazioni semantiche, via via sempre più svincolate da parametri oggettivi e sempre più correlate alle interpretazioni che i parlanti inferiscono dal contesto situazionale considerato ${ }^{3}$ :

Alla luce di tutto questo, dunque, è bene puntualizzare che la fase modale nel processo di grammaticalizzazione 
Al pasar del aspecto a la modalidad, entran en juego evaluaciones de tipo subjetivo, que nos llevan a interpretar la fase modal de la gramaticalización como posterior a la del aspecto, porque es sabido que los significados tienden universalmente a volverse menos dependientes de factores objetivos y más dependientes de las creencias y los juicios de los hablantes. (Melis 2006: 879)

Proprio da questi presupposti nascerebbe una delle valenze attualmente più associate alla perifrasi venir + gerundio, ossia la valenza approssimativa (RAE 2009: $550)^{4}$ :

(5) Lo que viene costando hoy en día un coche utilitario ( $>$ lo que cuesta aproximadamente)

In questo caso, non si parla di una singola automobile, bensì del prezzo approssimativo sul quale quel tipo di automobile si aggira attualmente.

Altri esempi di modalità approssimativa, in contesti diversi, sono offerti da Marcial Morera (1991: 243):

(6) Vienen siendo las cinco (= son las cinco, aproximadamente)

(7) Viene costando millón y medio (= cuesta aproximadamente un millón y medio)

Si tratterebbe dunque di una valenza tipicamente associabile a casi in cui l'ausiliare venir si combina con verbi di stato e in contesti nei quali interverrebbe un quantificatore (il quale, per l'appunto, segnalerebbe il valore cui tende l'approssimazione in questione).

Nello spagnolo attuale esiste un'altra perifrasi che è ampiamente impiegata per esprimere una valenza modale approssimativa del tutto analoga: si tratta di venir + $a+$ infinito.

\subsection{La perifrasi modale venir $+a+$ infinito}

Secondo Yllera (1980: 189-191), anticamente, in italiano, spagnolo e francese, questa perifrasi aveva valore expletivo: designava, cioè, un'azione che arriva al suo compimento. In spagnolo, particolarmente, poteva assumere sfumature analoghe a devenir. Nel caso dell'italiano, si tratta effettivamente della valenza anticamente attribuita alla perifrasi venire $a(d)$ essere, che tuttavia risulta essere sempre meno utilizzata. Torneremo su questo punto in $\S 3.2$.

Yllera (1980: 189-191) osserva che nel Poema de mio Cid ancora non si apprezzano occorrenze di questa perifrasi, benché venir sia già utilizzato con valenze strumentali che lo avvicinano al valore che assumerà come futuro ausiliare:

Si en el Cid no forma aún perífrasis de infinitivo, presenta ejemplos con una acepción cercana a la que toma en su empleo perifrástico. Venir significa "llegar a una situación o trance", vino a aquesto 112; "llegar a una acción”, la vin fer 3131; y

non ha decretato la fine o l'annullamento della fase aspettuale: i frutti di queste due fasi di evoluzione della perifrasi coesistono e si complementano nella molteplicità di valenze che essa stessa assume nella lingua attuale.

4 La valenza modale-approssimativa della perifrasi è segnalata anche da Gómez Torrego (1988: 168). 
con predicado del sujeto "venir a ser, hacerse", ricos son venidos todos los vassallos 1853 .

La comparsa della perifrasi vera e propria risalirebbe piuttosto al XIV secolo. Dal $\mathrm{XV}$ secolo in poi, si registra un aumento del suo uso associato a una valenza del tipo llegar $a . .$. / acertar $a . .$. , ossia con il valore expletivo già introdotto sopra.

Pare che la perifrasi esprima spesso un certo matiz de acción fortuita, che dunque si produce spontaneamente o in modo casuale, imprevisto. L'uso della perifrasi con questa valenza si confermerebbe a partire dal XV secolo.

Si tratta di un tipo di impiego di venir $+a+$ infinito che ancora si realizza nella lingua attuale, nei casi in cui il valore della perifrasi si avvicinerebbe a quello perfettivo di llegar $+a+$ infinito (García González 1992: 76) o di acabar + por + infinito (Gómez Torrego 1988: 104). Gómez Torrego (1999: 3383) osserva inoltre che la perifrasi, in determinati contesti, può imprimere al processo che designa una prospettiva di culminazione, di raggiungimento di una certa meta:

(8) El tiempo vino a darme la razón.

(9) Lo vino a saber por ti.

Secondo Melis (2006: 917-920), tuttavia, le — seppure innegabili — affinità deittiche tra l'ausiliare venir da un lato e llegar / acabar dall'altro sono limitate dal fatto che il primo ausiliare, contrariamente agli altri due, non designa un vero contatto col punto finale del processo azionale considerato. Ecco perché le perifrasi in questione, originariamente equivalenti in termini aspettuali, avrebbero poi iniziato a divergere nello sviluppo delle rispettive valenze modali.

Sarebbe appunto a partire dal XVII secolo che la perifrasi scatta ad una fase di grammaticalizzazione successiva, in cui affianca alla valenza aspettuale-perfettiva già commentata in precedenza una nuova valenza modale, tramite un'evoluzione che Gili Gaya (1943-1961: 94) designa in questi termini:

Del sentido terminativo que tiene la acción que viene a sin acabar de llegar ha nacido una expresión muy frecuente hoy, a la cual llamaremos aproximativa. Cuando decimos que un objeto viene a costar unas cincuenta pesetas significamos que, poco más o menos, cuesta esa cantidad, es decir, se acerca a ella sin que estemos seguros de si la alcanza o la rebaja ${ }^{5}$.

Ciò che emerge con forza, leggendo questa definizione di Gili Gaya, è il crescente grado di subjetivización che caratterizza questa valenza della perifrasi (Traugott 2001: 15), diretta manifestazione del salto da essa compiuto in termini di grammaticalizzazione. Come abbiamo visto anche per le valenze modali sviluppate da venir + gerundio, la soggettivizzazione consiste in quel processo tramite il quale i significati si rendono sempre più dipendenti dall'atteggiamento dell'osservatore nei confronti dell'enunciato (Melis 2006: 912). Si osservino gli esempi seguenti:

Molti sono gli studi che documentano questa valenza modale-approssimativa della perifrasi, tra cui Fente, Fernández y Feijóo (1976: 20), García González (1992: 77), Gómez Torrego (1988:104; 1999: 3383) e García Fernández (2006: 275). 
(10) El sustento que se nos daba... se reducía a una ganta (que viene a ser un almud) de arroz

(11) Separarlos y matarlos viene a ser lo mismo (Melis 2006: 919)

Di fatti, la valenza approssimativa è solo una tra quelle che discendono dall'incremento di questa tendenza a riferire una certa situazione sempre più in relazione alla percezione interiore dell'osservatore (soggettiva), e sempre meno in riferimento a parametri contestuali esterni (oggettivi).

Non a caso, la prima funzione che Olbertz (1998: 433-434) accorda a venir $+a+$ infinito è quella di mitigation device, utilizzato dal parlante per indicare che non si sente del tutto certo dell'appropriatezza di quanto sta affermando. Il ricorso alla perifrasi come elemento modalizzatore sarebbe dovuto all'esigenza del parlante stesso di tutelarsi in caso di disaccordo da parte del suo interlocutore. Ecco qualche esempio proposto dall'autrice:

(12) Todo esto ha venido a determinar la superpoblación de Madrid

(13) La compasión viene a ser el antídoto al suicidio

(14) Para muchísimos de los que residen en Madrid, teniendo las familias fuera, careciendo de hogar, el Ateneo viene a suplirlo en cierto modo (Olbetz 1998: 433434).

Olbertz osserva che non è sempre chiara quale parte dell'enunciato sarebbe oggetto di mitigazione-eufemizzazione, dato che l'uso della perifrasi con questa valenza si può constatare in un'amplissima gamma di contesti. Aggiunge però che venir $+a+$ infinito designa mancanza di precisione - avvicinandosi dunque alla valenza approssimativa già associata a venir + gerundio in $§ 2.2$. - solamente quando è presente un elemento quantificatore, come in:

(15) Viene a ser algo menos de siete octavos del total (Olbertz 1998: 434)

Molto spesso, proprio per enfatizzare la mancanza di precisione ${ }^{6}$ che lo spinge alla scelta della perifrasi come risorsa espressiva, l'interlocutore le abbina elementi ulteriormente relativizzanti, quali: una especie de, en cierto modo, más o menos, ecc. (Olbertz 1998: 434).

\subsection{Venir + gerundio o venir $+a+$ infinito? Una questione di approssimazione}

Da quanto si è osservato nei paragrafi precedenti, sembra che le due perifrasi si siano progressivamente accostate nell'espletare alcune funzioni di tipo modale; per lo meno, secondo la maggior parte degli studi consultati, esiste un generale accordo sul fatto che entrambe possono esprimere approssimazione quantitativa. Descrivendo venir + gerundio, Gómez Torrego (1988: 164) osserva: «Otro valor característico de esta perífrasis es el aproximativo. En estos casos, se pierde la noción de progresión

\footnotetext{
Tra gli autori che vedono questa perifrasi innanzitutto come una formula espressiva che designa dubbio o incertezza, risulta significativo includere anche Hernández Alonso (1984: 236-237), che la annovera tra le perifrasis dubitativas: «Adquiere un aspecto de imprecisión o aproximación. Nace este uso de una inseguridad en el valor terminativo que posee esta misma frase verbal: Las cosas vienen a ser más o menos como aqui»».
} 
de una acción verbal, y la perífrasis es equivalente a venir $+a+$ InFINITIVo». Secondo Torrego (1999: 3416): «[VENIR + GERUNDIO] puede tomar en ocasiones valor aproximativo, e. Este mueble viene saliendo por unas 50000 pesetas, pero en este caso es más frecuente VENIR + A + INFINITIVO ».

Questa supposta intercambiabilità tra le due perifrasi in contesti di approssimazione quantitativa sembrerebbe ormai assodata, nella lingua attuale.

Ma non mancano alcune perplessità, tra gli "addetti ai lavori": si tratta davvero di un'equivalenza totale? Ci sono eventuali elementi contestuali che potrebbero giustificare la scelta dell'una o dell'altra perifrasi?

Molto interessante, ad esempio, è il forum lanciato su questo tema all'interno del Foro del español del Centro Virtual Cervantes nel 20117: data la frase di partenza El billete sencillo viene costando unos 2 euros, i linguisti partecipanti dibattono nel forum sulla corretta interpretazione della perifrasi come espressione di posibilidad, duda, probabilidad o aproximación, in relazione alla possibile alternativa espressiva El billete sencillo viene a costar unos 2 euros.

L'opinione maggiormente condivisa nel forum, è che in El billete sencillo viene costando unos 2 euros l'approssimazione sarebbe data dall'elemento quantificatore unos. Di base, infatti, la perifrasi VENIR + GERUNDIO in sé, dato il suo eco aspettuale, designerebbe un'azione in via di sviluppo, tendente a un certo risultato / punto finale. Si tratta di una riflessione già proposta in relazione all'esempio (5): dobbiamo considerare l'elemento in questione (in quel caso, el precio de un coche utilitario; in questo, el precio del billete sencillo) come uno tra i vari esemplari di una serie che di esso si determinano nel tempo, a partire da un momento - precisato o meno - del passato per avvicinarsi a un certo valore di riferimento assunto nel momento di osservazione; è a tale valore, per l'appunto, che ci si approssima. La prova di tale interpretazione di venir + gerundio come perifrasi che abbraccia più tappe all'interno del processo azionale - come se si trattasse si sub-eventi di cui esso si compone - è data dalla possibilità di parafrasarla tramite l'avverbio últimamente. Parafrasi che, al contrario, non è possibile nel caso in cui si ricorra alla forma perifrastica venir a ser. Infatti, in El billete sencillo viene a costar unos 2 euros non si fa riferimento all'evoluzione di un prezzo - oggettiva - all'interno di un certo arco di tempo, quanto ad una stima dello stesso prezzo - soggettiva - nel momento di enunciazione. Questa formula sarebbe dunque quella più idonea a designare un'approssimazione intesa come frutto di una supposizione-ponderazione da parte del soggetto.

Queste osservazioni ci sono utili per comprendere come esista, di fatto, una differenza di prospettiva tra l'approssimazione teoricamente espressa da venir + gerundio e quella espressa da venir $+a+$ infinito. Tuttavia, si tratta di una differenza talmente sottile che i parlanti stessi non sembrano esserne realmente consapevoli. Come vedremo in $\S 4$ 4.1. e $\S 4.2$., di fatti, sono piuttosto comuni i casi in cui gli stessi nativi usano una delle due perifrasi nei casi in cui sarebbe preferibile l'uso dell'altra, e viceversa.

È opportuno sottolineare che, come si può constatare dagli esempi forniti in questo capitolo, questo parallelismo di valenza approssimativa tra venir $+a+$ infinito

Forum dal titolo Expresión de la aproximación y la duda; partecipanti al forum: Marcela Croitoru, Roberto Juan Beltrán Cortés, Piedad Zurita, Ángel Andrés Martínez Argos, Carlos Abrego, Ignacio Frías, Celia Sacristán Ferrari, Raul García; consultabile al seguente link: https:/cvc.cervantes.es/foros/leer_asunto1.asp?vCodigo $=40832$. 
e venir + gerundio si può identificare solo nei casi in cui l'ausiliare si abbina a una specifica classe azionale di verbi ausiliati, ossia quella dei verbi di stato ${ }^{8}$. È proprio su questi casi che abbiamo scelto di incentrare il presente studio.

\section{Venir siendo, venir a ser... e in italiano?}

Portiamo la nostra attenzione sui casi in cui venir + gerundio e venir $+a+$ infinito presentano il verbo essere come verbo ausiliato, come abbiamo visto negli esempi (4), (6), (10), (11), (13), (14) e (15).

Poniamoci ora la domanda: in lingua italiana esistono perifrasi corrispondenti a venir siendo e venir a ser?

\subsection{Venir siendo > venir essendo*}

Come osserva Barbagallo (2014:196), in italiano venire + gerundio si usa molto meno rispetto all'altra perifrasi continuativa (anch'essa, tuttavia, in calo sensibile nella lingua attuale) andare + gerundio; in effetti, presenta molte più limitazioni sul piano semantico, che riducono in modo consistente le categorie azionali ammesse dall'ausiliare. Tali rigide disposizioni normative del sistema italiano, puntualizza Barbagallo, impediscono una corrispondenza effettiva tra venire + gerundio e la corrispondente perifrasi spagnola.

La situazione attuale parrebbe una conseguenza del fatto che venire + gerundio sarebbe andata incontro ad un processo di grammaticalizzazione interrotta, che le ha impedito di sviluppare la stessa, ampia gamma di usi strumentali della corrispondente perifrasi spagnola; attualmente, di fatti, venire + gerundio sta progressivamente scomparendo dalla lingua italiana'. L'incremento dell'uso di altre perifrasi più grammaticalizzate — quali la polivalente stare + gerundio — avrebbe fatto il resto ${ }^{10}$.

Una delle più forti restrizioni azionali che caratterizzano venire + gerundio, nei casi in cui se ne può ancora apprezzare qualche occorrenza nella lingua attuale, ${ }^{11}$ è per l'appunto quella che sancisce la sua incompatibilità con i verbi di stato (Bertinetto 1991: 139).

\footnotetext{
La valenza assunta dalla perifrasi venir + gerundio, infatti, è strettamente vincolata all'aspetto lessicale del verbo ausiliato selezionato dall'ausiliare. Ad esempio, se l'ausiliato ha carattere dinamico, come nel caso dei verbi di attività -correre, parlare, camminare, ecc.- o compimento -costruire, scrivere, attraversare, ecc.- (Vendler 1967: 22), la perifrasi assumerà altre valenze quali la duratività o gradualità del processo designato, in risonanza con l'aspetto continuativo che permea la maggior parte dei suoi contesti d'uso. Sulle valenze aspettuali e azionali riconducibili a venir + gerundio, segnaliamo in particolare Martínez Atienza (2006: 268-272; 2017: 27-42) e Pippa (2006: 349-358)

9 Molto interessanti anche le riflessioni che propone in merito Brianti (2000: 102): «Al contrario, ANDARE + GERUNDIO, e VENIRE + GERUNDIO in modo ancora più marcato, sarebbero esempi di grammaticalizzazione interrotta (Giacalone Ramat 1995: 200), non solo per il calo della loro frequenza, ma anche per la loro specializzazione e idiomatizzazione. Essa si manifesta con la restrizione delle classi di aspetto lessicale dopo ANDARE e VENIRE e certe restrizioni morfologiche e lessicali su venire, che privilegia i tempi perfettivi e tende a fossilizzarsi in determinate forme, quali venir dicendo».

10 A tal proposito, si vedano gli studi di Durante (1981: 268-269) e Squartini (1998: 165).

11 Si tratta di occorrenze che rappresentano per lo più esempi di fossilizzazione, riscontrabili tendenzialmente nella lingua propria dei registri alti o dei linguaggi specialistici / lingue speciali (lingua letteraria, linguaggio giornalistico, scientifico, giuridico, ecc.; Giacalone Ramat 1995: 200).
} 
Per questa ragione, la realizzazione venir essendo* costituisce una forma agrammaticale: a dispetto della vicinanza formale rispetto alla spagnola venir siendo, dobbiamo dunque scartare necessariamente l'idea di una corrispondenza tra le due perifrasi.

\subsection{Venir a ser > venire a(d) essere}

Se già venire + gerundio risulta essere (ormai) poco presente, nei manuali e nelle grammatiche di lingua italiana attualmente disponibili, la perifrasi venire $+a+$ infinito quasi non vi compare. Di fatti, si tratta di una perifrasi molto scarsamente utilizzata in italiano, al punto che l'unico studio all'interno del quale si è potuto reperirne una qualche caratterizzazione è la Grammatica di Renzi, Salvi e Cardinaletti, nella quale Bertinetto (1991: 160-161) la include nella categoria delle perifrasi risolutive ${ }^{12}$ :

Queste perifrasi si collocano a parte, rispetto alle altre perifrasi verbali. (...)

Le perifrasi risolutive come finire per / venire fatto di (quest'ultima di stile arcaicizzante), e le altre citate qui di seguito, hanno la funzione di indicare il raggiungimento finale di un determinato risultato. Questo può essere frutto di uno sforzo intenzionale $\mathrm{o}$ (più frequentemente) rappresentare un esito inopinato.

(139) a. Dopo molti sforzi, gli venne fatto di centrare il bersaglio.

b. Senza quasi farci caso, finì per dirgli tutto.

Possono svolgere analogo ruolo perifrasi quali «venire / andare $a+$ infinito», «finire col + infinito», «andare a finire che + frase», ecc., talvolta caratterizzate da limitata applicabilità lessicale, e il più delle volte semanticamente trasparenti (cfr. anche 3.4.1):

(140) Il giavellotto andò / venne a cadere proprio ai piedi del giudice di gara.

(140) Per l'assenza di un'autentica programmazione, la casa finì coll'avere un solo servizio.

(141) Ogni volta andava a finire che lui se ne ricordava troppo tardi.

Dato il prevalente carattere non intenzionale di queste perifrasi, l'imperativo è escluso. Di difficile impiego è anche l'accezione progressiva dei Tempi imperfettivi.

Secondo questa prospettiva, dunque, la perifrasi non porrebbe tanto l'accento sull'intenzionalità da parte del parlante circa un fine da raggiungere, quanto piuttosto sul raggiungimento circostanziale del fine stesso (Amenta / Strudshlom 2002: 14).

Una fonte di informazione supplementare su questa perifrasi, ormai piuttosto latitante nelle grammatiche e nei manuali di lingua, si può ancora rinvenire in alcuni dizionari di lingua italiana, alla voce venire. Lo Zingarelli (2019: 2561-2562), ad esempio, propone queste due valenze: «n.2 Dire cose inaspettate, singolari / con va-

12 Si tratterebbe della perifrasi prospettiva «dovere + infinito» e delle perifrasi risolutive «finire per + infinito» e «venire fatto di + infinito». 
lore intens.: Ma che mi vieni a raccontare? [...] n.10 (fam.) "trovarsi a essere": Lui viene a essere suo cognato».

Nel Grande dizionario italiano dell'uso (De Mauro et al., 2000: 987-988) troviamo numerose locuzioni verbali che coinvolgono il verbo venire, quali: venire a capo, venire a conoscenza, venire a contesa, venire a galla, venire ai fatti, venire al bisogno, venire al dunque, venire alla conclusione, venire alla luce, venire in mente, ecc. Ciascuna di queste locuzioni pone l'accento su una data prospettiva a livello di perfettività, la quale è appunto la valenza di fondo comune a tutte le locuzioni annoverate. Si cita solo una realizzazione sul modello venire $+a+$ infinito, ossia venire a sapere, come sinonimo di venire a conoscenza, anch'essa espressione dalla pronunciata valenza non intenzionale. Gli stessi risultati si trovano anche alla voce venire del Dizionario etimologico Zanichelli (Cortelazzo e Zolli 1988: 1422-1423).

Il Grande dizionario di Italiano Garzanti (AA.VV. 2008: 2749), oltre a riproporre le locuzioni verbali già viste nei dizionari precedenti, analizza la perifrasi venire a costare: «costare complessivamente: tra stoffa e manifattura verrà a costare complessivamente quattrocento euro; quanto viene? (fam.) quanto costa? $\left.{ }^{13}\right\rangle$.

Tra i pochi dizionari che presentano qualche riferimento più generale alla forma venire $+a+$ infinito si può citare il Devoto-Oli (2009: 3068), che associa alla perifrasi una valenza del tutto in linea con quella di tipo perfettivo proposta da Bertinetto (1991: 161):

n.18: Con valore fraseologico, raggiungere un certo risultato o una data situazione, giungere (con la preposizione $a$ e l'inf.): venni a sapere che lo zio era morto; mi sono venuto a trovare in una situazione senza uscita; sono venuto a credere che sia meglio vendere il negozio.

Altro dizionario che riconosce questa valenza di venire $+a+$ infinito è il Sabatini Coletti (2008: 2996): «In unione con la prep. a seguita da inf., esprime il raggiungimento di un certo risultato: Sono venuto a sapere certe cose!; Si vennero a trovare in un grosso guaio; quanto viene a costare l'appartamento?». Il fatto che venire + $a+$ infinito, nella maggior parte dei dizionari consultati, sia analizzata in relazione a specifiche valenze contestuali — e solo abbinata ad un numero limitato di verbi principali — si rispecchia anche nella scarsa diffusione nell'uso di questa perifrasi nella lingua attuale: sembra che, di fatto, essa sopravviva solo in questi pochi usi idiomatizzati che ne citano i dizionari.

Tuttavia, diversamente da quanto abbiamo visto per venire + gerundio, venire + $a+$ infinito ammette il verbo essere come ausiliato. Il valore che assume la perifrasi in questo caso è quello perfettivo proposto da Bertinetto (1991: 161), lo stesso che emerge anche nei dizionari che propongono qualche esempio per gli usi di venire $a(d)$ essere che abbiamo osservato: la perifrasi sembra voler inquadrare il processo designato in una prospettiva nella quale $i$ fatti si producono spontaneamente, in modo incidentale o comunque non interferito dal soggetto.

Si tratterebbe di un valore perfettivo veicolabile anche dalla corrispondente spagnola venir a ser (Gómez Torrego 1999: 3383), analizzato in §2.3., negli esempi (8) e (9).

13 In questa valenza della perifrasi possiamo riconoscere una certa corrispondenza con il valore approssimativo (quantitativo) della spagnola VENIR A COSTAR analizzato da Olbertz (1988: 434). 
Non a caso, come osserva Yllera (1980: 189), sia l'italiano, che il francese e lo spagnolo hanno mutuato una propria perifrasi a partire dalla forma romanza venire $a d+$ infinito, caratterizzata da un valore essenzialmente expletivo, che designava per l'appunto una acción que llegaba a producirse. Lo spagnolo moderno, spiega Yllera, avrebbe poi sviluppato ulteriori valenze associabili alla perifrasi venir a ser, come quella effettiva (vino a ser jefe) o quella approssimativa (el argumento de la peli viene a ser el que os he contado), pur continuando ad associarla, se il contesto lo richiede, anche a una valenza di tipo accidentale.

L'italiano sembrerebbe dunque essere rimasto piuttosto vincolato al valore originale della perifrasi venire $(a d)$ essere, che non è giunta a sviluppare la stessa varietà di valenze della forma spagnola corrispondente.

\section{Dallo spagnolo all'italiano: un test... approssimativo}

\subsection{Il test: item, obbiettivi e gruppo di informanti}

Da quanto si è argomentato fino ad ora possiamo trarre le seguenti conclusioni a livello contrastivo, tra spagnolo e italiano:

a. venir siendo non è traducibile con *'venire essendo';

b. venir a ser, grammaticalmente parlando, sarebbe traducibile con 'venire ad essere'; tuttavia, la perifrasi italiana risulta essere scarsamente utilizzata nella lingua attuale; inoltre, l'unico valore che può presentare, in comune con venir a ser è un valore di tipo perfettivo; al contrario, non può esprimere alcun valore di tipo modale-approssimativo.

Giunti a questo punto, ci chiediamo: qual è la reazione di un madrelingua italiano nei confronti delle perifrasi venir siendo e venir a ser, utilizzate in contesti di tipo approssimativo. Gli risulteranno tutto sommato trasparenti? Potrà decifrare correttamente la valenza da esse veicolata, in base al contesto dato? $\mathrm{E}$, infine, come le tradurrebbe nella sua lingua madre?

Per raccogliere qualche dato reale in risposta a questi interrogativi, si è creato questo breve test, composto di 5 item:

TEST

\begin{tabular}{|l|l|}
\hline Quanti anni hai? & \\
\hline $\begin{array}{l}\text { Qual è la tua lingua madre? (se ne hai } \\
\text { più di una, indicale tutte) }\end{array}$ & \\
\hline Da quanto tempo studi lo spagnolo? & \\
\hline $\begin{array}{l}\text { Hai mai avuto esperienze di soggiorno } \\
\text { in territori ispanofoni? }\end{array}$ & \\
\hline $\begin{array}{l}\text { Se la risposta alla domanda precedente } \\
\text { è positiva, indica in che paese, e per } \\
\text { quanto tempo }\end{array}$ & \\
\hline
\end{tabular}


CONSEGNA: Di seguito, troverai vari frammenti di testo in lingua spagnola (tratti da diverse fonti online: quotidiani, forum, siti internet di varia tipologia). Scrivi la traduzione in lingua italiana SOLO DEI PASSAGGI IN CORSIVO in ogni item. Durante la prova, NON potrai ricorrere all'uso di dizionari, né alla consultazione di risorse online. Buon lavoro!

1) La última película de un Dani de la Orden, que no hay que confundir (en nuestras cabecitas locas) con Dani de la Torre es menos sólida, menos eficiente, y menos divertida que la mucho más saludable El pregón, en donde el estupendo reparto conseguía levantar lo que aqui se queda a medio gas tanto por desinterés, como por falta de interés en intentar cualquier otra cosa que no sea lo que viene a ser, así, lo que es: Nada del otro mundo. Ni tan mal, ni tan bien, que da un poco lo mismo.

2) Las 10 nuevas palabras de la RAE que más nos han sorprendido (pero que muy mucho).

TÁPER - Que significa: recipiente de plástico con cierre hermético, que se usa para guardar o llevar alimentos. Lo que viene siendo el táper de toda la vida del Señor, vamos.

3) También uno de los mejores discos de electrónica experimental de la década, Third Law, de Roly Porter, viene a ser un intento de descifrar a través de la música la complejidad del cosmos.

4) El proyecto de colaboración entre la Biblioteca Eugenio Trías-Casa de Fieras del Retiro (Madrid) y el Hospital Universitario Infantil Niño Jesús "La lectura que da vida" lleva esperanza y evasión, en forma de libros, a menores pacientes de Oncología y Psiquiatría, niños que por su delicado estado de salud no pueden acceder a la biblioteca y a los servicios que este centro bibliotecario muy próximo al hospital ofrece (...).

«Sinceramente es un proyecto irresistible desde donde se mire. No se trata únicamente de los niños y adolescentes, pues vienen siendo unos 75 al mes, también debemos considerar a las familias y al personal sanitario en su totalidad, como participes y animadores a su vez».

5) Sin salir del puerto y en dirección sur, nos encontramos con lo que viene a ser la plaza mayor de Liverpool, Albert Dock. Con una estructura rectangular, este lugar alberga restaurantes y tiendas pero, a diferencia de la plaza mayor de Madrid y al estar en el puerto, en el centro hay agua.

I 5 item del test riportano occorrenze della perifrasi venir siendo o venir a ser reperite in documenti di vario tema e registro, disponibili in rete: blog, siti di critica cinematografica, quotidiani online, pagina web del Gobierno de España ${ }^{14}$. In ciascuno degli item proposti, le due perifrasi coinvolte esprimono una certa valenza approssimativa.

Si è chiesto ai candidati di tradurre dallo spagnolo all'italiano la frase dell'item nella quale apparivano, di volta in volta, le due forme in questione. Per fornire loro qualche elemento in più che li supportasse nell'interpretazione delle due perifrasi, è stata inclusa negli item parte del contesto che le accompagnava nel testo-fonte.

Inoltre, nell'item 5 si è scelto di includere un ulteriore elemento al quale queste perifrasi risultano essere spesso abbinate, nello spagnolo corrente.

Attualmente, si riscontra una certa tendenza ad utilizzare queste perifrasi abbinate al pronome que preceduto dall'articolo neutro lo - a designare il soggetto dell'azione in questione _ - in casi in cui il loro uso non sarebbe necessario a livello

\footnotetext{
Le fonti da cui sono stati estrapolati i testi degli item sono consultabili nella sitografia finale.
} 
sintattico. Come una sorta di muletillas lingüísticas ${ }^{15}$, ossia di riempitivi cui appoggiarsi in termini espressivi; Come osserva Domingo Argüelles (2018: 222; 360), le varianti più ricorrenti in cui si impiegherebbe questo riempitivo sarebbero lo que es e lo que sería, benché, come sostiene l'autore, non manchino i casi in cui esso si abbina a perifrasi, come in lo que viene siendo o lo que viene a ser:

Propio de locutores y comentaristas de la radio y la televisión que han hecho escuela en España y en México, entre otros países hispanohablantes, la perifrasis lo que viene siendo es un pendejismo que surgió como muletilla en el habla y que se ha incrustado en la lengua escrita de un modo espantoso. Como los locutores no pueden dejar espacios vacíos en tanto parlotean, recurren a mil muletillas y lugares comunes que repiten incansablemente. (...) La explicación de Moreno de Alba no debería dejar lugar a dudas. ¿Qué sentido tiene decir, como lo hacen los reporteros de la radio y la televisión, Hay muchos damnificados en lo que es el Estado de Jalisco o en lo que viene siendo el Estado de Jalisco? Hay muchos damnificados en Jalisco, y punto. Todo lo demás es superfluo y ridículo. No aporta nada a la comunicación y retuerce la lengua horriblemente, tal como la han de tener quienes usan dichas expresiones.

L'esempio di Moreno de Alba citato da Domingo Argüelles, di fatto, è rappresentativo di molti casi in cui la combinazione di lo que, con le perifrasi venir siendo e venir a ser, non solo non apporta alcun plus di significato, ma sortisce - spesso a insaputa del parlante - l'effetto opposto, offuscando e diluendo eccessivamente quanto sta comunicando ${ }^{16}$.

L'obiettivo di questa prova era per l'appunto quello di raccogliere qualche reazione reale da parte di nativi italiani che venissero in contatto con queste 5 circostanze comunicative in cui venir siendo e venir a ser sono utilizzate con valenza modale approssimativa: attraverso le loro interpretazioni, ci si proponeva di misurare:

- $\quad$ fino a che punto cogliessero le sfumature modali espresse dalle due perifrasi, caso per caso;

- raccogliere — data l'impossibilità di procedere per una traduzione meramente formale, come si è specificato in $\S 3$. - qualche proposta concreta per una resa di queste valenze in lingua italiana.

È una prova che propone documenti dalle tipologie testuali e dai registri linguistici piuttosto vari, e che coinvolge strutture perifrastiche la cui corretta interpretazione modale - come si è visto in $\S 2.4$. - è talvolta talmente complessa da generare dubbi perfino tra linguisti di alto livello.

15 «muletilla.(Del dim. de muleta). 1. f. muleta (de los toreros). 2. f. Voz o frase que se repite mucho por hábito». (DRAE 2014: 1509).

16 Come puntualizza Domingo Argüelles nel capitolo 151 del suo libro, è tuttavia importante tenere a mente che esistono, beninteso, casi nei quali è corretto l'uso di lo que es / lo que sería: «Evidentemente hay muchísimos enunciados en los cuales la expresión lo que es está correctamente empleada. Existen incluso contextos gramaticales que ayudan a explicar por qué está bien usada y por qué no puede eliminarse. Esto sucede, por ejemplo, cuando tiene como antecedente una oración completa o la palabra todo: no cumplió su palabra, lo que es inaceptable; la multiplicación tan fácil de todo lo que es negativo; o cuando está precedida de una preposición y seguida de un adjetivo o adverbio: hay una ignorancia total en relación con lo que es ético y conveniente». Si tratta certamente di spunti utili circa l'uso corretto di questa forma linguistica - ormai in rapida espansione - sulla quale è tuttavia auspicabile che in un futuro prossimo la ricerca linguistica dedichi ulteriori approfondimenti. 
Un esercizio, dunque, in cui saper trarre il massimo supporto orientativo dal limitato contesto messo a disposizione per ogni item diviene essenziale per poter interpretare correttamente la valenza delle perifrasi coinvolte. Per tutti questi motivi, si è scelto di sottoporre la prova a un gruppo di informanti avvezzi a lavorare in condizioni linguistiche di questo tipo, ritenendoli i più idonei a fornire risposte interessanti agli interrogativi in questione: si è selezionato un gruppo di 12 studenti del corso di Traduzione specialistica italiano-spagnolo I e II del Corso di Laurea Specialistica in Traduzione della Scuola Superiore di Lingue Moderne per Interpreti e Traduttori (SSLMIT-Università degli Studi di Trieste) $)^{17}$.

\subsection{Risultati}

Gli item in cui gli informanti hanno avuto meno difficoltà di decodifica sono il 4, il 5 (più o meno allo stesso livello) e 1'1.

Il testo dell'item 4 è tratto dalla descrizione del progetto La lectura que da vida, a favore dei pazienti minorenni ricoverati nel reparto di psicologia e oncologia dell'Hospital Universitario Infantil Niño Jesús, di Madrid. Si tratta di un articolo pubblicato nel sito internet del Ministerio de Cultura y Deporte del governo spagnolo. Pertanto, il registro che contraddistingue questo item è più alto-formale rispetto, per esempio, a quello degli item 1, 2 e 3.

La perifrasi venir siendo, in questo caso, è accompagnata dal complemento di quantità 75 , dunque esprime approssimazione quantitativa, in linea con l'uso che si è analizzato in El billete sencillo viene costando unos 2 euros. Sembra che l'approssimazione di tipo quantitativo veicolata da questa perifrasi sia la più trasparente, per i candidati del test: la presenza di un elemento quantificatore, associato ad un contesto ben definito come quello di questo item, permette loro di optare, senza eccessiva esitazione, per soluzioni traduttive che rispecchiano un'approssimazione di questo tipo. Essi propongono dunque soluzioni che prevedono l'uso dell'avverbio circa, l'avverbio che esprime probabilmente per antonomasia l'idea di approssimazione in lingua italiana, associato a verbi all'indicativo presente in forma sintetica (se ne calcolano, sono-x8-, ne arrivano); uno dei candidati propone anche l'uso della locuzione avverbiale approssimativa alla fin fine.

Il testo dell'item 5 è tratto dal blog Ave dónde voy, dedicato al tema dei viaggi. Si è scelto di introdurre un estratto del testo in cui si apprezza un uso di (con) lo que viene a ser come riempitivo. Ci troviamo in tal caso in una situazione del tutto analoga a quella evocata da Domingo Argüelles (2018) in § 4.1., tramite l'esempio Hay muchos damnificados en lo que viene siendo el estado de Jalisco: In questo

17 La prova è stata svolta da 12 studenti in tutto, nell'aprile del 2019. Tramite le informazioni a carattere socio-linguistico fornite da ogni informante, raccolte attraverso la tabella di apertura del test, si è constatato che: c'erano 9 informanti di 23 anni, 2 di 24 e 1 di 25; tutti i candidati erano nativi italiani; essendo tutti studenti del corso di Laurea Specialistica in Traduzione, avevano già tutti -per la struttura del percorso formativo pervista dalla SSLMIT- un livello di spagnolo LS piuttosto alto, con almeno 4 anni di studio di questa lingua alle spalle; tuttavia, molti di loro hanno dichiarato di averne ben di più: 5 ( 3 candidati), 8 ( 2 candidati) 9 ( 1 candidato) o addirittura 10 (ben 4 candidati); A livello di soggiorni in territorio ispanofono, solo un paio di candidati avevano avuto un'esperienza di Erasmus della durata di 5 mesi, gli altri candidati avevano per lo più avuto qualche sporadica e breve occasione di viaggio in Spagna; in ogni caso, si tratta di parentesi di immersione nella lingua piuttosto limitate nel tempo, che probabilmente non hanno inciso in modo sensibile sui risultati della prova in oggetto. I candidati, durante il test, non hanno avuto la possibilità di consultare manuali o dizionari di spagnolo, né di consultare altre tipologie di risorse online. 
item, l'apporto della formula con lo que viene a ser la plaza Mayor de Liverpool, in termini di significato è pari a zero: il senso della frase è, semplicemente, nos encontramos con la Plaza Mayor de Liverpool. In generale, i candidati hanno (correttamente) interpretato la struttura en lo que viene a ser come un riempitivo, proponendo complessivamente due tipi di soluzione: alcuni l'hanno neutralizzato, evitando di trasporlo nella loro risposta (ci troviamo di fronte/davanti alla piazza [...]; possiamo notare la piazza $[\ldots]$; ci imbattiamo nella piazza [...]); cinque candidati, invece, hanno risposto ricorrendo a una formula riempitiva analoga in italiano (ci imbattiamo / troviamo in quella che è [...] ci troviamo davanti a quella che è [...]; ci trovammo in quella che è [...]).

Il testo dell'item 1 è tratto da un breve articolo online, che si presenta come una piccola recensione - dal registro piuttosto informale - sul film El mejor verano de mi vida. Nel contesto all'interno del quale si inserisce, la valenza che esprime questa perifrasi è di tipo approssimativo ad ascendenza per così dire perfettiva, richiamando quella riconoscibile in acabar + por + infinito, secondo quanto abbiamo visto nell'epigrafe $\S 2.3$. Possiamo anche percepire nell'uso della perifrasi il ricorso al mitigation device (Olbertz 1998: 434-435), presentato in $\S 2.3$., che tuttavia, in questo caso, sarebbe in secondo piano, come ad integrare la sfumatura perfettiva. Sfumatura che per la maggior parte degli informanti risulta piuttosto trasparente: lo possiamo leggere attraverso le soluzioni traduttive che essi propongono, in cui non di rado intervengono parole che richiamano una certa perfettività del processo designato: occorrenze del verbo diventare (diventa, è diventato), sostantivo risultato o occorrenze del verbo risultare (portasse a un risultato, risulta essere, risulta essere).

I 2 item in cui gli informanti sembrano aver riscontrato maggiore difficoltà di decodifica sono il 2 e il 3.

Il testo dell'item 2 è tratto da un forum online in cui si proponeva un piccolo excursus sulle nuove parole entrate tra i lemmi del dizionario RAE nel 2017. Tra questi, si disquisiva su táper, adattamento dell'inglese tupper. La perifrasi viene siendo, in questo caso, assume una valenza approssimativa piuttosto simile a quella presente nell'item 1 . In realtà, le perifrasi coinvolte nei due item in questione sono diverse, ma - come abbiamo visto in $\S 2.4$. — spesso si riscontrano nella lingua attuale casi di sconfinamento di una delle due perifrasi nel terreno di "competenza modale" dell'altra: in questo caso, venir siendo assume una valenza approssimativa con funzione relativizzante che teoricamente - in assenza di un elemento quantificatore - sarebbe piuttosto propria di venir a $\mathrm{ser}^{18}$. Pare che questo abbia generato qualche perplessità in termini interpretativi, possibilmente accentuata dal fatto che vari informanti sembrano non essere del tutto sicuri sull'interpretazione dell'espressione colloquiale de toda la vida del Señor, qui utilizzata per indicare familiarità. Gli elementi che trasmettono in modo più evidente un'idea di approssimazione, nelle loro risposte, sarebbero l'uso dell'avverbio insomma, o eventualmente l'uso del condizionale in sarebbe il solito tupperware, che smorza l'assertività che pur tuttavia caratterizza l'indicativo usato nel resto delle risposte raccolte. In questo caso, insomma, la resa della modalità approssimativa espressa da viene siendo sembra in qualche modo carente nelle traduzioni proposte, il che fa supporre che forse non sia stata colta correttamente nella perifrasi di partenza.

\footnotetext{
Vale a dire, una valenza analoga a quella che abbiamo visto per venir a ser nell'esempio (11).
} 
Giungiamo infine all'item 3, nel quale alla massima presenza del mitigation device nel contesto di partenza corrisponde massima opacità di resa modale nel contesto di arrivo.

Il testo di questo item è tratto da un articolo online di el Periódico-Arte. Nel passaggio proposto, l'autore dell'articolo utilizza appunto la perifrasi viene a ser come espediente espressivo per conferire relatività a quanto sta affermando, una sua personale interpretazione sul disco in questione. La maggior parte delle soluzioni traduttive proposte dai candidati denotano invece un grado di assertività piuttosto alto: la maggior parte di loro opta per l'uso di verbi in forma sintetica al presente indicativo - rappresenta, si presenta, risulta, è considerato, si rivela -, arrivando a

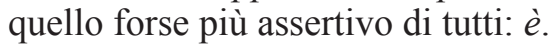

Soluzioni di questo tipo non rispecchiano in modo tangibile l'uso di viene a ser come espediente relativizzante-eufemizzante, valenza che, probabilmente, i candidati non riconoscono nell'uso della perifrasi spagnola in contesti di questo tipo. $\mathrm{La}$ maggior parte dei candidati propone una traduzione piuttosto discostata dalla forma perifrastica di partenza; solo uno di loro opta per una traduzione parzialmente letterale, arriva ad essere, che tuttavia non risulta del tutto soddisfacente: non trattandosi di una perifrasi utilizzata nell'italiano attuale, suona più come un calco dal testo di partenza.

Per una lettura in chiave più globale dei risultati di questo test, abbiamo ritenuto opportuno avvalerci del grafico illustrativo riportato qui di seguito, che potemmo definire un grafico "a opacità crescente":

(figura 1)

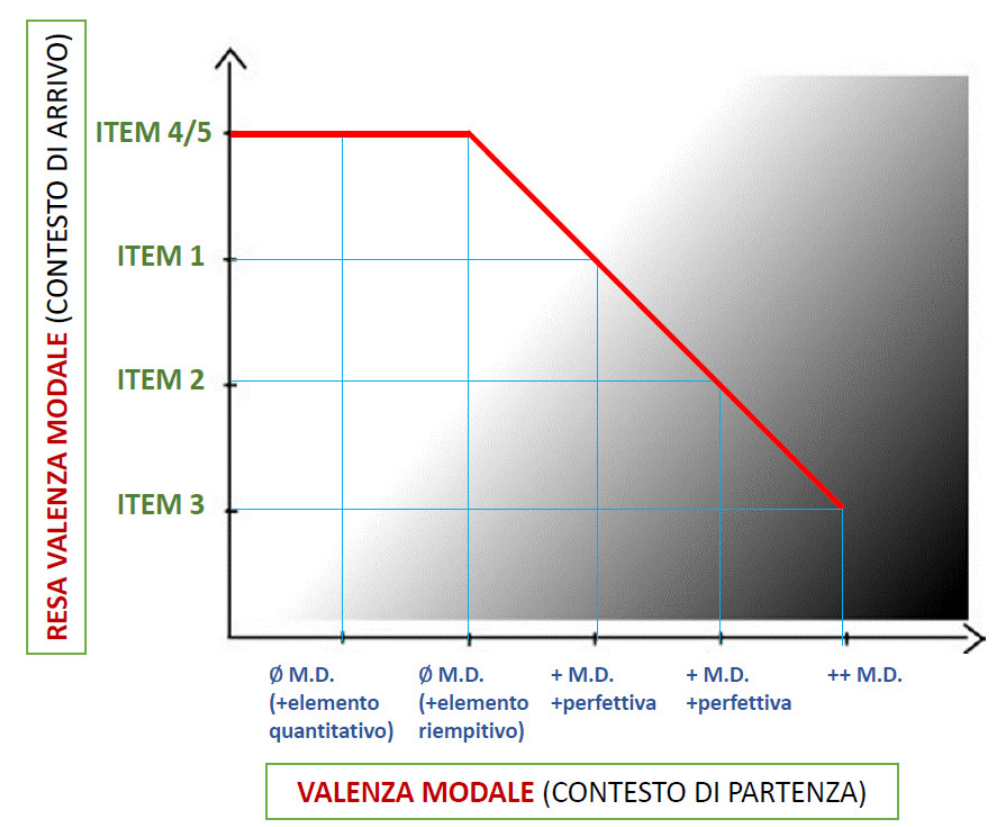

Le ordinate dell'asse verticale del grafico sono rappresentate dai vari item del test: al livello più alto si posizionano gli item la cui valenza modale espressa dalle 
perifrasi venir a ser / venir siendo risulta essere più efficacemente trasposta nelle proposte traduttive dei candidati: item 4 e 5; procedendo verso il basso, si trovano invece gli item per i quali le proposte traduttive dei candidati si rivelano via via sempre meno adeguate nel riflettere appieno la valenza modale della perifrasi spagnola di partenza: item 1, item 2 e item 3;

L'asse orizzontale del grafico segnala invece, in progressione da sinistra a destra, la presenza di una valenza modale di tipo relativizzante (mitigation device) ${ }^{19}$ all'interno degli item osservati: come si è già osservato, negli item 4 e 5 tale carica è pressoché nulla, in quanto sono altre le funzioni modali o retoriche espletate dalle perifrasi coinvolte; negli item 1 e 2 , la valenza relativizzante è presente ma non preponderante, in quanto si combina in entrambi i casi ad una valenza di tipo perfettivo; nell'item 3 , infine, la carica modale veicolata dalle perifrasi è totalmente relativizzante.

Se incrociamo gli item dell'asse verticale con il livello dell'asse orizzontale che identifica la proporzione della carica relativizzante in essi contenuta, otteniamo una serie di punti, che se congiunti delineano una traiettoria discendente. La tonalità di fondo del grafico degrada progressivamente dal bianco al nero, man mano che si procede dall'angolo superiore sinistro all'angolo inferiore destro: tramite questo cromatismo, il grafico ci mostra che l'efficacia traduttiva delle proposte risulta essere inversamente proporzionale alla presenza della componente relativizzante nella perifrasi di partenza. Ecco perché gli item il cui valore modale è risultato più facilmente decifrabile per gli informanti del test sono segnalati dai punti più vicini all'angolo superiore sinistro del grafico, mentre quelli più "opachi" in termini tanto di interpretazione quanto di resa in lingua d'arrivo si avvicinano progressivamente all'angolo più oscuro dello stesso, quello inferiore destro, l'apice di opacità modale.

Il punto forse più interessante che emerge da questi risultati, è proprio quello secondo cui gli item risultano tanto più opachi per gli informanti quanto più si intensifica la carica modale del mitigation device espressa dalla perifrasi di partenza. In tali termini, non sarebbe un caso che siano proprio gli item in cui le perifrasi non veicolano tale valenza modale (item 4 e 5) a suscitare meno difficoltà interpretativa e traduttiva, difficoltà che invece aumenta quando si constata una presenza del mitigation device in combinazione ad altre valenze modali (item 1 e 2) o meno (item 3 ).

I risultati del test ci confermano anche che, allo stadio di sviluppo diacronico attuale, la lingua italiana non presenta perifrasi che si possano considerare (e utilizzare) come corrispondenti di venir siendo e venir a ser, in contesti di modalità approssimativa. Lo dimostra il fatto che i candidati non ricorrono mai - salvo in un caso, tra le soluzioni traduttive proposte per l'item 4 - all'uso di venire $a(d)$ essere come alternativa traduttiva di venir a ser.

Questo dato deporrebbe dunque a favore della tesi per cui questa perifrasi è ormai inutilizzata nella lingua attuale, per lo meno nell'italiano di uso medio correntemente utilizzato dai nativi nella maggior parte dei contesti comunicativi quotidiani. Allo stesso modo, ovviamente, i candidati optano per una necessaria riformulazione negli item 2 e 4, dovendo scartare la possibilità di tradurre viene/n siendo con le corrispondenti (e agrammaticali) perifrasi italiane viene / vengono essendo*.

Nella figura 1, mitigation device è indicato tramite la sigla MD. 


\section{Conclusioni}

Il percorso che abbiamo costruito tramite questo studio ci ha portato ancora una volta a toccare con mano le divergenze - spesso insospettabili - che caratterizzano in molteplici casi il rapporto tra italiano e spagnolo. L'affinità tra queste due lingue, come ben sa chi le studia (e ancor più chi le insegna), infatti, non deve mai essere presa sottogamba.

Nel caso delle perifrasi venir siendo e venir a ser, nella fattispecie, abbiamo constatato come l'espressione della modalità approssimativa (o forse, alla luce di quanto esposto, dovremmo dire delle modalità approssimative) sia frutto della moltiplicazione delle valenze modali assunte dalle due perifrasi nella lingua attuale.

Valenze spesso talmente sottili e talmente liquide, da non poter essere attribuite con esclusività né all'una né all'altra perifrasi.

Come orientarsi, allora, per una loro corretta interpretazione? La chiave, in questo come in molti altri casi, è data dal contesto comunicativo.

Non a caso, una linea di ricerca sviluppatasi negli ultimi decenni promuove un approccio di spirito semantico-pragmatico allo studio delle perifrasi, che analizza la relazione tra i cambi di significato dell'ausiliare e i contesti d'uso, accordando una speciale attenzione alle inferenze che scaturiscono, presso i parlanti, dall'associazione di determinate perifrasi a specifici elementi del discorso: tali inferenze, una volta divenute sufficientemente stabili nel tempo, potrebbero a loro volta convertirsi in parte del nuovo significato della perifrasi; esse, infatti, vincolerebbero forme esplicite e significati occulti coesistenti all'interno dello stesso messaggio (Traugott / König 1991: 211).

Tali presupposti divengono ancora più importanti nella prospettiva di un apprendente di spagnolo italofono: in mancanza di due perifrasi italiane corrispondenti a venir siendo e venir a ser, il suo sforzo dovrà essere maggiore, tanto in fase di decodificazione della valenza modale approssimativa da esse veicolata, quanto nella ritrasmissione quanto più fedele possibile di quella stessa valenza in lingua italiana.

In questo senso, il docente avrà una doppia responsabilità nei confronti dell'apprendente: da un lato, dovrà impegnarsi ad offrigli un contatto il più ampio e diversificato possibile con contesti comunicativi in cui le perifrasi spagnole in questione esprimano approssimazione, accompagnandolo alla scoperta dei loro significati caso per caso; dall'altro lato, consapevole dell'indisponibilità di perifrasi italiane ad esse corrispondenti, dovrà guidarlo nella scelta di soluzioni linguistiche altrettanto adeguate, per forma e fruibilità a livello modale, in lingua italiana.

\section{Riferimenti bibliografici}

Amenta, Luisa / Strudshlom, Erling (2002): «"andare a + infinito" in italiano. Parametri di variazione sincronici e diacronici», Cuadernos de Filología Italiana, 9, pp. 11-29.

Barbagallo, Simona (2014): «Estudio contrastivo de algunas perífrasis de gerundio en español e italiano», Annali della facoltà di scienze della formazione, 13, pp. 179-197.

Bertinetto, Pier Marco (1991): «Il verbo», in Lorenzo Renzi, Giampaolo Salvi, Anna Cardinaletti (a c. di), Grande Grammatica italiana di consultazione, Bologna il Mulino, vol. 2, pp. 13-161. 
Brianti, Giovanna (2000): «Diacronia delle perifrasi aspettuali dell'italiano: Il caso di "stare" + gerundio, "andare" e "venire" + gerundio», Lingua Nostra, 61, pp. 35-52; 97-120.

Camus Bergareche, Bruno (2004): «Perífrasis verbales y expresión del aspecto en español», in Luis García Fernández, Bruno Camus Bergareche (a c. di), El pretérito imperfecto, Madrid, Gredos, pp. 511-572.

Dietrich, Wolf (1973 [1983]): Der periphrastische Verbalaspekt in den romanischen Sprachen: Untersuchungen zum heutigen romanischen Verbalsystem und zum Problem der Herkunft des periphrastischen Verbalaspekts, Tübingen, Niemeyer. [Trad. sp. di H. M. Martínez, El aspecto verbal perifrástico en las lenguas románicas: Estudios sobre el actual sistema verbal de las lenguas románicas y sobre el problema del origen del aspecto verbal perifrástico, Madrid, Gredos, 1983].

Domingo Argüelles, Juan (2018): Las malas lenguas. Barbarismos, desbarres, palabros, redundancias, sinsentidos y demás barrabasadas, Ciudad de México, Editorial Océano.

Durante, Marcello (1981): Dal latino all'italiano moderno. Saggio di storia linguistica e culturale, Bologna, Zanichelli.

Fente Gómez, Rafael / Fernández Álvarez, Jesús / Feijóo, Lope G. (1976): Perífrasis verbales, Madrid, PBE- S.G.E.L.

García Fernández, Luis / Carrasco Gutiérrez, Ángeles / Martínez Atienza, María (2006): Diccionario de perifrasis verbales, Madrid, Gredos.

García González, Javier (1992): Perifrasis verbales, Madrid, S.G.E.L.

Giacalone Ramat, Anna (1995) «Sulla grammatizzazione dei verbi di movimento "andare” e "venire" + gerundio», Archivio glottologico italiano, 80, pp.168-203.

Gili Gaya, Samuel (1943-1961): Curso superior de sintaxis española, Barcelona, Vox / Bibliograf.

Gómez Torrego, Leonardo (1988): Las perifrasis verbales, Madrid, Arco Libros.

Gómez Torrego, Leonardo (1999): «Las perífrasis verbales de infinitivo», in Ignacio Bosque, Violeta Demonte (a c. di), Gramática descriptiva de la lengua española, Madrid, Espasa Calpe, vol. 3, pp. 3323-3389.

Hernández Alonso, César (1984): Gramática Funcional del español, Madrid, Gredos.

Martínez-Atienza, María (2006): «Venir + gerundio», en L., García Fernández et al. (a c. di), Diccionario de perifrasis verbales, Madrid, Gredos, pp. 268-272.

Martínez-Atienza, María. (2017): «Venir + gerundio del español, venire + gerundio del italiano y otras perífrasis verbales», Sintagma: revista de lingüística, 29, p. 27-42. https:// doi.org/10.21001/sintagma.2017.29.02.

Melis, Chantal (2006): «Verbos de movimiento. La formación de los futuros perifrásticos», en Concepción Company Company (a c. di), Sintaxis histórica de la lengua española. Primera parte: La frase verbal, Universidad Nacional Autónoma de México, Fondo de Cultura Económica, vol. 2, pp. 875-968.

Morera Pérez, Marcial (1991): Diccionario crítico de las perifrasis verbales del español, Puerto del Rosario, Servicio de Publicaciones del Cabildo Insular de Fuerteventura.

Musto, Salvatore / Ripa, Valentina (2005): «Le perifrasi gerundivali in spagnolo e in italiano: uno studio contrastivo», in M. C. Ruta, L., Silvestri (a c. di), Actas XXIII Congreso AISPI, Palermo, Flaccovio editore / Centro Virtual Cervantes, pp. 408-427.

Olbertz, Hella (1998): Verbal Periphrases in a Functional Grammar of Spanish. Berlin /New York, Mouton de Gruyter, "Functional grammar series" 22.

Pippa, Salvador (2006): «Venir, vir, venire + gerundio nella perifrasi continua: quale traduzione verso l'italiano?» in Graziano Benelli, Giampaolo Tonini (a c. di), Studi in ricordo di Carmen Sánchez Montero, Trieste, EUT Edizioni, pp. 349-358 
RAE (2009) = Real Academia Española, Nueva gramática de la lengua española, Madrid, Espasa Calpe.

Sánchez Montero, Ma Del Carmen (1993): Perífrasis verbales en español e italiano. Estudio contrastivo, Trieste, Edizioni Lint.

Spaulding, Robert K. (1926): History and Syntax of the Progressive Constructions in Spani$s h$, Berkeley, University of California Press.

Squartini, Mario (1998): Verbal Periphrases in Romance: Aspect, Actionality, and Grammaticalization, Berlin / New York, Mouton de Gruyter.

Traugott Closs, Elisabeth (2001): «Historical Pragmatics», in Laurence R. Horn, Gregory Ward (a c. di), Handbook of Pragmatics, Oxford, Blackwell, pp. 538-561.

Traugott Closs, Elisabeth / König, Ekkehard (1991): «The semantics-pragmatics of grammaticalization revisited», in Elisabeth Traugott, Bernhard Heine (a c. di), Approaches to Grammaticalization, Amsterdam/Philadelphia, Benjamins, vol. 1, pp. 189-218.

Vendler, Zeno (1967): Linguistics in Philosophy, Ithaca, Cornell University Press.

Yllera Hernández, Alicia (1980): Sintaxis histórica del verbo español: las perífrasis medievales, Zaragoza, Universidad de Zaragoza.

\section{Dizionari}

AA.VV. (2008): Grande Dizionario di Italiano, Milano, Garzanti.

Cortelazzo, Manlio / Zolli Paolo (1988): Dizionario etimologico della lingua italiana, vol. 5 ( S-Z), Bologna, Zanichelli.

De Mauro, Tullio / Lepschy, Giulio C. / Sanguineti, Edoardo (2000): Grande dizionario italiano dell'uso, Torino, UTET.

DRAE (2014): Diccionario de la lengua española, Madrid, Espasa Libros S.L.U., $23^{\mathrm{a}}$ ed.

Devoto, Giacomo / Oli, Gian Carlo (2009): Il Devoto - Oli Vocabolario della lingua italiana, a c. di Luca Serianni e Maurizio Trifone, Milano, Mondadori Education.

Sabatini, Francesco / Coletti, Vittorio (2008): Il Sabatini Coletti. Dizionario della lingua italiana, Milano, Rizzoli Larousse.

Zingarelli, Nicola (2019): Lo Zingarelli. Vocabolario della lingua italiana, a c. di Mario Cannella, Beata Lazzarini, Bologna, Zanichelli.

\section{Altre fonti citate: sitografia}

Centro Virtual Cervantes-Archivo del Foro del Español (2012). «Expresión de la aproximación y la duda», <https://cvc.cervantes.es/foros/leer_asunto1.asp?vCodigo $=40832>$ [08/07/2011]

\section{Sitografia item test}

ITEM 1: Paires Iglesias, Juan (2018): «El mejor verano de mi vida» - Como quien tiene una pecera en casa. elseptimoarte.net, <https:/www.elseptimoarte.net/noticias/criticas/el-mejor-verano-de-mi-vida---como-quien-tiene-una-pecera-en-casa-33494.html> [11/07/2018].

ITEM 2: Forum Goodgamesestudios (2018): Don Burro, <https://community.goodgamestudios.com/empire/es/discussion/147305/don-burro/p18> [23/05/2018].

ITEM 3: García Montoliu, Álvaro (2019): Basinski hace música con la colisión entre dos 
agujeros negros, elperiódico.es, <https:/www.elperiodico.com/es/port/arte/20190305/ album-musico-william-basinski-incorpora-sonido-colision-agujeros-negros $>$ [05/03/2019].

ITEM 4: Ministerio de Cultura y Deporte - Gobierno de España: <https:/www.culturaydeporte.gob.es/cultura/areas/libro/mc/lectureando/lectureando-con/biblioteca-eugeniotrias. html> [29/01/2020].

ITEM 5: Ave Dónde Voy - blog (2017): Manchester y Liverpool en dos días, $<$ https://avedondevoy.blogspot.com/2017/04/manchester-y-liverpool-en-2-dias.html? spref=pi> [12/04/2017]. 